\title{
INTERCORRÊNCIAS GRAVÍDICAS E RESULTADOS DO TESTE DO REFLEXO VERMELHO EM RECÉM-NASCIDOS
}

\author{
*Airton Arison Rêgo Pinto I \\ Fabíola Chaves Fontoura II \\ Adriana Lorrayny Barboza Pereira Ramos III \\ Lucas Ramos da Costa IV
}

\section{RESUMO}

As intercorrências gravídicas trazem riscos não somente para a gestante, mas também para o público infantil, como as alterações oculares, constituindo-se o Teste do Reflexo Vermelho como aliado para a detecção precoce. O estudo objetivou descrever as intercorrências gravídicas apresentadas pelo público materno durante o período gestacional e associá-las aos resultados do Teste do Reflexo Vermelho nos recém-nascidos. Esta pesquisa partiu de um estudo descritivo, de abordagem quantitativa, realizada na Maternidade Almeida Castro, em Mossoró, Rio Grande do Norte. A amostra foi constituída por 79 mães e seus recém-nascidos, tendo como instrumento de coleta de dados um formulário estruturado com perguntas objetivas. A coleta de dados deu-se entre os meses de fevereiro a setembro de 2018, sendo posteriormente analisados a partir da frequência absoluta e relativa, e para a análise da associação entre as variáveis intercorrências gravídicas e o resultado do Teste do Reflexo Vermelho, aplicou-se o teste qui-quadrado. Após a análise dos resultados, do público estudado composto por 79 mães, pertencentes exclusivamente ao município de Mossoró-RN, 49 apresentaram intercorrências, com maior prevalência da Infecção do Trato Urinário. Os resultados dos testes nos respectivos recém-nascidos mostraram reflexo vermelho normal, não apresentando associações significantes entre as intercorrências maternas e os resultados do teste do reflexo vermelho nos recém-nascidos, com $p=0,99$. As intercorrências gravídicas, de acordo com o estudo, não interferiram nos resultados dos testes, porém deve-se cuidar das intercorrências apresentadas pelas mulheres durante a gestação, a fim de minimizar os potenciais riscos à saúde ocular dos fetos.

PALAVRAS-CHAVE: Saúde Ocular. Complicações na Gravidez. Neonato.

Enfermeiro. Especialista em Educação Continuada e Permanente em Saúde. Faculdade de Enfermagem Nova Esperança, FACENE. Departamento de Enfermagem. CEP: 59625080, Mossoró, Rio Grande do Norte, Brasil.*Autor correspondente: airton.arison17 Orcid: 0000-0001-6800-425X

Enfermeira. Doutora em Enfermagem. Faculdade de Enfermagem Nova Esperança, FACENE. CEP: 59609700, Mossoró, Rio Grande do Norte, Brasil. Orcid: 0000-0002-5254-526X

Enfermeira. Especialista em Saúde Pública e Programa Saúde da Família para Enfermeiro. Faculdade de Venda Nova do Imigrante. CEP: 29375-000, Santa Cruz, Espírito Santo, Brasil. Orcid: 0000-0002-0825-5564

Agrônomo. Doutor em Manejo de Solo e Água. Faculdade de Enfermagem Nova Esperança, FACENE. Departamento de Agonomia. CEP: 59628608, Mossoró, Rio Grande do Norte, Brasil. Orcid: 0000-0002-8710-1589 


\section{INTRODUÇÃO}

O Ministério da Saúde (MS) deixa claro que todas as mulheres em período gestacional têm o direito de fazer, no mínimo, seis consultas de pré-natal, no intuito de garantir uma gestação com saúde e uma parturição tranquila. No entanto, é perceptível deficiências do acompanhamento pré-natal, tendo como possíveis consequências, as intercorrências gravídicas. ${ }^{1}$ Neste sentido, é preciso realizar, durante as consultas de prénatal, o rastreamento de fatores que possam afetar a saúde materna e, consequentemente, a saúde ocular do feto, como a pré-eclâmpsia, eclâmpsia e de infecções como, por exemplo, a toxoplasmose, a sífilis e a rubéola. ${ }^{2}$

O próprio MS propõe que os profissionais enfermeiros desenvolvam no seu dia a dia o acompanhamento do crescimento e desenvolvimento infantil; incentivo e orientação quanto ao aleitamento materno; diagnóstico de enfermagem e planos de cuidados em relação as doenças prevalentes na infância, como também na realização da triagem neonatal (Teste do Olhinho, Pezinho, Orelhinha, Coraçãozinho e Linguinha). ${ }^{3}$

O exame detalhado do olho, que está incluso no exame físico geral, é de competência do enfermeiro, ${ }^{4}$ tanto em crianças como em adultos, visto que esse procedimento não dispõe de riscos e é de extrema importância para a manutenção da saúde ocular. ${ }^{5}$ Sabese que saúde ocular é essencial, pois a visão do ser humano constitui um dos sentidos mais importantes, logo contribui para desenvolvimento psicoemocional, físico e cognitivo. ${ }^{6}$ Porém, a perda significativa da visão não antecede queixas e observa-se que na infância, durante o período escolar, a visão tende a suprir bem a necessidade destas. ${ }^{7}$

Em estados de agravamento da visão, pode-se desenvolver a cegueira infantil mesmo que dados apresentem ser algo raro, pois se estima que apenas $4 \%$ da população mundial infantil tenham cegueira, ou seja, correspondem numericamente a 14 milhões de crianças. ${ }^{8,9}$

Solebo, Teoh e Rahi ${ }^{8}$ mostram em seu estudo uma redução da cegueira infantil ocasionada por doenças infecciosas como, por exemplo, a rubéola e o próprio sarampo.

Por essa razão, o teste do reflexo de Bruckner, conhecido como teste do olhinho ou Teste do Reflexo Vermelho (TRV), é uma maneira pela qual se pode detectar possíveis alterações visuais, funcionando como importante auxiliador para os profissionais de enfermagem, como medida de prevenção a cegueira infantil, detectando precocemente as lesões oculares, o que contribui no desenvolvimento de intervenções precisas e resolutivas. ${ }^{2}$

Desta forma, os profissionais podem se preparar para atender a população materna, durante o período gestacional, realizando um pré-natal de qualidade e com uma visão mais crítica para as implicações das intercorrências gravídicas para a saúde das gestantes e, consequentemente, a saúde ocular dos neonatos e crianças. Contudo, o presente estudo objetivou descrever as intercorrências gravídicas apresentadas pelo público materno, durante o período gestacional, e associá-las aos resultados do Teste do Reflexo Vermelho em recém-nascidos. 


\section{MATERIAL E MÉTODOS}

Tratou-se de um estudo descritivo, com abordagem quantitativa, realizado na Maternidade Almeida Castro, no município de Mossoró, Rio Grande do Norte. Maternidade referência em assistência ao recém-nascido saudável e de risco. Essa maternidade, consta de Unidades de Cuidados Intensivos e Intermediários Neonatais, Unidade de Cuidados Intermediários Canguru, Alojamento Conjunto, Salas de Partos, entre outros setores.

A população foi constituída por mães e recém-nascidos que estavam internados na referida maternidade. Compuseram a amostra por conveniência 79 mães e os seus recémnascidos que realizaram o Teste do Reflexo Vermelho (TRV), independentemente da idade gestacional do neonato, avaliado pelo método de Capurro Somático: sexo, peso, comprimento, tipo de parto, além da mãe ter realizado o pré-natal, tendo feito no mínimo 3 consultas e apresentarem resultados de exames laboratoriais realizados durante a gestação. Além disso, ter aceitado participar da pesquisa e assinado o Termo de Consentimento Livre e Esclarecido (TCLE). Foram excluídas mães com deficiência auditiva e visual devido a dificuldade de comunicação com os pesquisadores.

\section{RESULTADOS E DISCUSSÃO}

Diante da amostra de 79 mães, 62\% (49) referiram ter tido uma ou mais intercorrências durante a gestação, sobressaindo diante daquelas que não possuíram nenhum tipo, com 38\% (30) das mulheres. Comparando com estudo realizado num instituto de referência ao atendimento de gestantes no estado do Rio de Janeiro, as mulheres classificadas com gestação de alto risco, um percentual de $20,3 \%$ apresentou intercorrências e 45,2\% constou alguma afecção obstétrica. ${ }^{10}$ Assim,
VOLUME 18 - NÚMERO 2 - Ago/2020 ISSN ELETRÔNICO 2317-7160

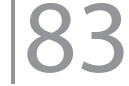

Os dados foram coletados a partir de um formulário previamente estruturado, com perguntas objetivas para obtenção dos dados quantitativos sobre as variáveis maternas (existência e tipos de intercorrências gravídicas apresentadas) e neonatal (resultados do TRV), aplicados durante o período de fevereiro a setembro de 2018 .

Os resultados foram organizados em planilhas no programa Excell, versão 2010 e processados no programa Statistical Package for the Social Sciences (SPSS) versão 22.0, a partir de uma análise quantitativa, além de serem expressos em frequência absoluta e relativa.

Para a análise da associação entre as intercorrências gravídicas e o resultado do TRV, aplicou-se o teste Qui-Quadrado ( $\chi 2)$, mostrando aquelas análises que apresentaram um nível de significância de $5 \%$, ou seja, quando o valor de $\mathrm{p}<0,05$.

A pesquisa foi aprovada pelo CEP da instituição proponente: Faculdade de Enfermagem e Medicina Nova Esperança FAMENE/FACENE - PB, com número CAAE: 67775517.7.0000.5179 e com parecer de $n^{\circ}$ 2.057.781 em 11 de maio de 2017. vê-se que existe aproximação da porcentagem mensurada pela pesquisa com o estudo, mostrando que as intercorrências gravídicas se fazem presentes em quantidade significativa de mulheres no período gestacional, independentemente do tamanho amostral em cada pesquisa.

Dentre as 49 mães que apresentaram uma ou mais intercorrências gravídicas, conforme a Figura 1, a Infecção Urinária obteve um percentual de $58 \%$ (41), ou seja, 
esta infecção se destacou em relação as demais citadas pelas mães, seguidas do Sangramento com $10 \%$ e Hipertensão com $7 \%$ da amostra. Dentre as Infecções Sexualmente
Transmissíveis (IST's) citadas pelas mães, foram encontradas: candidíase e sífilis, com percentual de $2 \%$ (1) de acometimento.

FIGURA 1: Intercorrências Gravídicas das mães de recém-nascidos que realizaram o Teste do Reflexo Vermelho. Mossoró - Rio Grande do Norte. Brasil, 2018, n=49

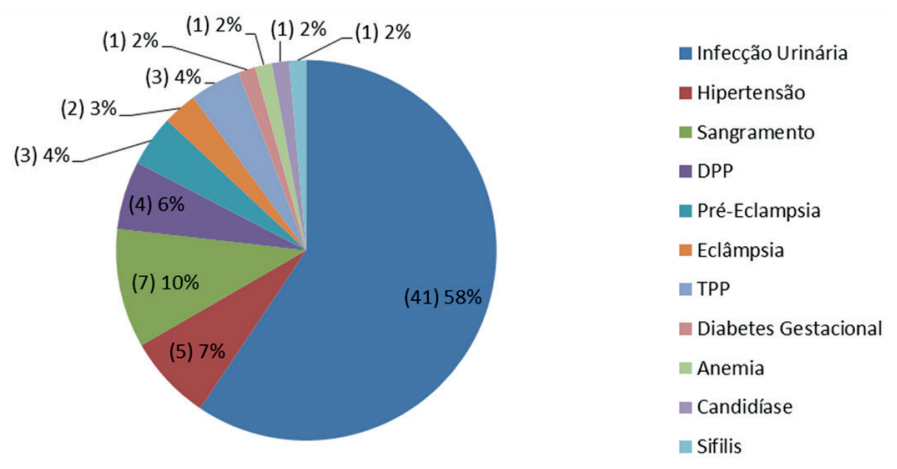

Fonte: Dados da pesquisa direta, Mossoró, Rio Grande do Norte 2018

A Infecção do Trato Urinário (ITU) foi identificada em laudos de 137 (25,5\%) mulheres em período gestacional dentro de uma amostra de 538 uroculturas realizadas na cidade de Veranópolis, Rio Grande do Sul.11 Esta infecção é encontrada comumente em mulheres durante a gestação e pode acarretar problemas relacionados ao desenvolvimento fetal, como Amniorrexe prematura (rotura prematura das membranas ovulares/RPMO), ${ }^{12}$ a Diabetes Mellitus Gestacional (DMG) ${ }^{13}$ e outras patologias existentes que podem alterar a saúde ocular dos recém-nascidos.

Outra infecção de grande impacto na saúde da mulher é a sífilis, principalmente no período gestacional, podendo acometer o recém-nascido, conhecida como sífilis congênita. Esta por sua vez pode trazer problemas para saúde dos recém-nascidos deixando sequelas em nível físico, no desenvolvimento e também sensoriais e, quando acometidos gravemente, podem ser fatais ao feto. ${ }^{14}$ Já Peixoto, ${ }^{15}$ em sua revisão de literatura traz a Candidíase como um relevante problema de saúde pública uma vez que de 70 a $75 \%$ das mulheres em geral, em idade reprodutiva, apresentam um caso de candidíase durante essa fase de suas vidas.

Em relação ao teste do reflexo vermelho, a literatura enfatiza sua aplicação como meio importante na detecção de possíveis alterações e o grau de relevância para a prevenção de cegueira infantil muitas vezes evitável, que resultam na impossibilidade de visualizar o reflexo ou até mesmo referir a qualidade deste reflexo. ${ }^{16}$ Sabe-se que a aplicabilidade desse Teste é algo simples e prático de ser realizado e que não sofre interferências quanto ao local de sua realização como, por exemplo, na rotina das Unidades Básicas de Saúde, apesar da particularidade de cada recém-nascido ou criança avaliada. Além disso, um estudo que avaliou a saúde ocular através do TRV em Unidade Básica de Saúde, as mães questionadas demostraram total interesse sobre a saúde ocular dos seus filhos, de forma receptiva, indagativas e extremamente atentas sobre as considerações da avaliação realizada. ${ }^{17}$

Ao verificar possíveis associações 
estatísticas entre as intercorrências

recém-nascidos, a Tabela 1 apresenta o valor gravídicas e os resultados dos TRV dos

do $p$.

TABELA 1: Associação entre intercorrências na gestação e o resultado do Teste do Reflexo Vermelho. Mossoró - Rio Grande do Norte. Brasil, 2018, $n=49$.

\begin{tabular}{ccccccc}
\hline \multirow{2}{*}{ Variável } & \multicolumn{3}{c}{ Grupo de mães } & \multicolumn{2}{c}{ TRV do RN } & \multirow{2}{*}{ p valor } \\
\cline { 2 - 4 } Intercorrências Gravídicas & 49 & 100 & 49 & 100 & 0,99 \\
\hline \hline & Freq. & $\%$ & Freq. & $\%$ & \\
\hline
\end{tabular}

A conclusão de todos os Testes de Reflexo Vermelho, realizados nos recémnascidos, resultaram "normais". Após correlacionar as intercorrências gravídicas e os resultados dos testes de reflexo vermelho nos recém-nascidos, percebeu-se que não houve associação estatística significante, pois 0 valor de $p=0,99$ foi superior a 0,05.

A exemplo de alterações oculares, pesquisa feita com 32 recém-nascidos, 30 destes tiveram como resultado do TRV "normal" totalizando $94 \%$ da amostra analisada e apenas dois apresentaram quadro "suspeito" tendo como reflexo a cor acinzentada ou com ausência de brilho (opaca). ${ }^{18}$ Outro estudo sobre aplicação do TRV em 123 recém-nascidos internados em uma UTI Neonatal, apenas dois tiveram alteração, mas lesão visual foi descartada pelo oftalmologista. ${ }^{19}$

Na Turquia, pesquisa feita com 1.358 recém-nascidos, 800 estavam internados em alojamento conjunto e 558 na UTI Neonatal, sendo que $7(0,88 \%)$ e $14(2,51 \%)$ recém- nascidos, respectivamente, apresentaram TRV anormal. Além disso, verificou-se associação estatística significante ao associar a variável "casamento consanguíneo" com a anormalidade do reflexo vermelho, sendo valor de $p=0,01720$, comprovando que, além de intercorrências durante a gestação, o fator consanguíneo contribui para possíveis alterações oculares no feto.

A participação e colaboração das mães é de fundamental importância, pois os fatores apresentados pelo público materno, como doenças infecciosas, durante o período gestacional (toxoplasmose, citomegalovírus e rubéola), e também dos fatores da história neonatal (baixo peso, baixo quantidade de oxigênio no sangue e a situação da prematuridade) podem estar relacionados com o reflexo vermelho. Por meio da investigação, através do teste, se faz algum tipo de associação comprovando a existência de vários fatores que são vistos como sendo de riscos para a saúde ocular, trazendo possíveis alterações na visão dos recém-nascidos. ${ }^{2}$

\section{CONCLUSÃO}

Os resultados revelaram que, dentre as intercorrências apresentadas pelas gestantes, sobressaíram as infecções urinárias, sangramento e hipertensão, sendo que estas não implicaram em alterações oculares nos recém-nascidos, de acordo com a associação estatística para a amostra estudada.

Entretanto, faz-se necessário que 
as gestantes realizem um pré-natal eficaz, a fim de identificar possíveis alterações e/ou intercorrências no período gravídico, para a realização do tratamento precoce e assim reduzir os possíveis danos à saúde ocular nos recém-nascidos.

\title{
PREGNANT INTERCURRENCES AND RESULTS OF THE NEWBORN RED REFLEX TEST
}

\begin{abstract}
Pregnancy complications pose risks not only for pregnant women, but also for the newborn, such as eye changes, with the Red Reflex Test as an ally for early detection. The study aimed to describe the pregnancy complications presented by the pregnant women during the gestational period and to associate them with the results of the Red Reflex Test in newborns. This research started from a descriptive study, with a quantitative approach, carried out at Almeida Castro Hospital, in Mossoró, Rio Grande do Norte. The sample consisted of 79 mothers and their newborns at the maternity hospital, using a structured form with objective questions as their data collection instrument. Data collection took place between the months of February to September 2018, being subsequently analyzed from the absolute and relative frequency, and for the analysis of the association between the pregnancy complications variables and the result of the Red Reflex Test, it was applied to the chi-square test. After analyzing the results, of the studied public of 79 mothers belonging exclusively to the municipality of Mossoró-RN, 49 presented complications, with a higher prevalence of Urinary Tract Infection. The test results in the respective newborns showed a normal red reflex, with no significant associations between maternal complications and the results of the red reflex test in the newborns, with $p=0.99$. Pregnancy complications, according to the study, did not interfere with the results of the tests, but care should be taken with the complications presented by women during pregnancy, in order to minimize the potential risks to the ocular health of fetuses.
\end{abstract}

KEYWORDS: Eye Health. Pregnancy Complications. Newborn.

\section{REFERÊNCIAS}

1. Neves ACF. Principais dificuldades em acompanhar as gestantes pela equipe de saúde da família. UFMG. [Principais dificuldades em acompanhar as gestantes pela equipe de saúde da família]. Faculdade de medicina, Araçuari; 2010;35p.

2. Aguiar ACS, Cardoso MVLML, Lúcio IML. Teste do reflexo vermelho: forma de prevenção à cegueira na infância. Rev. Bras. Enferm. 2007; 60 (5): 541-05.
3. Brasil. Ministério da Saúde. Secretaria de Atenção à Saúde. Departamento de Ações Programáticas Estratégicas. Diretrizes de Atenção à Saúde Ocular na Infância: detecção e intervenção precoce para prevenção de deficiências visuais/Ministério da Saúde, Secretaria de Atenção à Saúde, Departamento de Ações Programáticas Estratégicas. - Brasília: Ministério da Saúde. 2013; [número de páginas aproximado 40p]. 
4. Cofen. Portal do Cofen - Conselho Federal de Enfermagem. Resolução Cofen-358/2009. Disponível em: http://www.cofen.gov.br/ resoluo-cofen-3582009_4384.html.

5. Coren/RS - Conselho Regional de Enfermagem do Rio Grande do Sul. Parecer técnico Nº 05/2016 - Análise sobre a realização de teste de reflexo vermelho pelo enfermeiro. 2015. Disponível em: https://www.portalcorenrs.gov.br/docs/ Pareceres/Parecer_ctue_ctsab_052016.pdf.

6. BRASIL. Ministério da Saúde. Secretaria de Atenção à Saúde. Diretriz de Atenção à Saúde Ocular na Infância: detecção e intervenção precoce para prevenção de deficiências visuais. 2.ed. Brasília, 2016; 45p. Disponível em: http://bvsms.saude.gov.br/bvs/publicacoes/ diretrizes_saude_ocular_infancia_prevencao_ deficiencias_visuais.pdf.

7. Iqbal S, Klein B. Approach to acute vision loss in children. Uptodate. 2019; 36(3): 264-07.

8. Sobelo AL, Teoh L, Rahi J. Epidemiology of blindness in children. Archives Of Disease In Childhood. 2017; 102(9): 853-04.

9. Chong C, Mcghee CNJ, Dai SH. Causes of childhood low vision and blindness in New Zealand. Clin. Experiment. Ophthalmol, 2019; 47(2): 165-05.

10. Xavier RB, Jannotti CB, Silva KS, Martins AC. Risco reprodutivo e renda familiar: análise do perfil das gestantes. Ciênc. Saúde Coletiva. 2013; $18(4): 1161-10$.

11. Pancotto C, Von Ameln OL, Cattani F. Perfil de resistência, etiologia e prevalência de patógenos isolados em uroculturas de gestantes atendidas em um laboratório de análises clínicas da cidade de Veranópolis, Rio Grande do Sul. RBAC. 2019; 51(1): 29-33.
12. Portela FM, Marcolan, LB, Reis AA, Lucca DPP, Filho JKP, Rosas LEFCL, et al. Infecção urinária como fator de risco para amniorrexe prematura. Rev Cad de Med. 2019; 02(03):85-93.

13. Paraná. Secretária de Estado de Saúde do Paraná. Superintendência de Atenção à Saúde. Linha guia de diabetes mellitus/SAS. 2. ed. Curitiba: SESA, 2018; 12p.

14. BeckEQ, Souza MHT. Práticas de enfermagem acerca do controle da síflis congênita. Rev. Pesqui. Cuid. Fundam. 2018; 10(3): 19-24.

15. Peixoto JV. Candidíase -uma revisão de literatura. Brazilian Journal of Surgery and Clinical Research, Ipatinga. 2014; 2(8): 75-82.

16. Tuli SY, Giordano BP, Kelly M, Fillipps D, Tuli SS. Newborn with an absent red reflex. J Pediatr Health Care. 2013; 27(1): 51-5.

17. Mussavi M, Asadollahi K, Janbaz F, Mansoori E, Abbasi N. The evaluation of Red Reflex sensitivity and specificity test among neonates in different conditions. Iran J Pediatr. 2014; 24(6): 697-02.

18. Rodrigues ÉC, Cardoso MVLL, Aguiar ASC, Cezario KG. Resultado do Teste Reflexo Vermelho em recém-nascidos. Rev Enferm. UFPE. 2018; 12(2): 433-8.

19. Dorsch FLB, Marvilha MHR, Passamani RPS, Frauches DO. Alterações ao teste do reflexo vermelho em recém-nascidos internados na unidade de terapia intensiva de um hospital filantrópico em Vitória/ES, Brasil. Rev Bras Pesqui Saúde. 2016; 18(3): 49-57.

20. Ozkurt Z, Balsak S, Yildirim Y, Yuksel H, Caca I. Associations between red reflex abnormality, consanguinity and intensive care hospitalization of newborns in Turkey. East Mediterr Health J. 2018; 24(7): 631-6. 